\title{
Bed-side Open Tracheostomy in COVID-19 patients: Our experience on a safe and swift operative approach
}

\author{
Nur Wahidah Wahid ${ }^{1}$, Peter Deutsch ${ }^{2}$, Aakash Amlani $^{3}$, Huw Griffiths $^{4}$, and Ijaz Ahmed ${ }^{4}$ \\ ${ }^{1}$ Health Education England West Midlands \\ ${ }^{2}$ The Royal Wolverhampton Hospitals NHS Trust \\ ${ }^{3}$ Birmingham Heartlands and Solihull (Teaching) NHS Trust \\ ${ }^{4}$ Heartlands Hospital
}

September 21, 2020

\begin{abstract}
5 Key points: * As an aerosol-generating procedure (AGP), tracheostomy poses significant viral transmissions risks to health care workers * The decision about tracheostomy method and optimal setting depends on a multitude of local factors with no available studies to suggest the superior option * We describe our effective, safe and swift approach to bedside open tracheostomy during the COVID-19 pandemic * A key approach in performing an effective bedside tracheostomy is to ensure that the procedure is standardised and performed by highly experienced surgeons, anaesthetist and scrub team. * Our experience with bedside open tracheostomy demonstrated a short mean procedural time, with no tracheostomy-related complications and no reported viral transmission amongst the healthcare members involved.
\end{abstract}

Title: Bed-side Open Tracheostomy in COVID-19 patients: Our experience on a safe and swift operative approach

Article type: Correspondence - Our Experience

\section{Key points:}

- As an aerosol-generating procedure (AGP), tracheostomy poses significant viral transmissions risks to health care workers

- The decision about tracheostomy method and optimal setting depends on a multitude of local factors with no available studies to suggest the superior option

- We describe our effective, safe and swift approach to bedside open tracheostomy during the COVID-19 pandemic

- A key approach in performing an effective bedside tracheostomy is to ensure that the procedure is standardised and performed by highly experienced surgeons, anaesthetist and scrub team.

- Our experience with bedside open tracheostomy demonstrated a short mean procedural time, with no tracheostomy-related complications and no reported viral transmission amongst the healthcare members involved.

Key words: Tracheostomy, Covid-19, SARS-CoV-2, Aerosol-generating procedure

\section{Background}

The COVID-19 global pandemic has caused an increased number of patients requiring prolonged mechanical ventilation and subsequently requiring tracheostomy for weaning of mechanical ventilation. Aerosolgenerating procedures (AGP) such as intubation and tracheostomy poses significant viral transmissions risks 
to healthcare workers. A systematic review evaluating transmission of acute respiratory infection to health care workers during the SARS outbreak in 2003 estimated odds of transmission from tracheostomy and intubation of OR 4.2 and OR 6.6 respectively (1). Several tracheostomy guidelines have emerged worldwide which have provided invaluable input including international multidisciplinary guidance (2), and society guidance by ENT UK (3), and British Laryngology Association (4), amongst others.

Tracheostomy can be performed as an open surgical procedure, percutaneous, or hybrid. The decision about the optimal location for a tracheostomy procedure depends on a multitude of local factors with no available studies to suggest the superior option. The purpose of this article is to share our approach to performing bedside surgical tracheostomy in COVID-19 patients in a safe and effective manner, whilst minimising the risk of viral transmission.

\section{Methods}

As recommended by ENT UK, we established a COVID Airway Team within the ENT department at [removed for blind peer review], consisting of four highly experienced head and neck consultant surgeons. The choice of tracheostomy method and setting was agreed on a case-by-case basis via a multi-disciplinary approach. Bedside surgical tracheostomy was the preferred option particularly in patients that were deemed at higher risk for instability during transfer. Our aim is to perform bedside surgical tracheostomy with maximum efficiency and safety, over the shortest time possible.

The procedure was planned as a semi-elective to ensure availability of the most experienced head and neck surgeons, senior anaesthetists and highly-skilled and familiar scrub team, whilst maintaining a minimum safe number of personnel present. No more than two surgeons per procedure were involved. Team members are all equipped with appropriate personal protective equipment (PPE) in accordance with World Health Organisation (WHO) and Public Health England (PHE) guidance. A delegated PPE officer ensures that PPE donning and doffing are performed by healthcare members effectively. All necessary equipment was prepared by experienced theatre nurse with reference to a standardised checklist, and set up according to the layout demonstrated in Figure 1. Figure 2 highlights further descriptions of our bedside open surgical tracheostomy technique, based on recommendations by ENT UK and British Laryngological Society.

\section{Results}

During the COVID-19 period between $1^{\text {st }}$ March 2020 and $1^{\text {st }}$ June 2020, 30 patients with COVID-19 underwent a tracheostomy at our institution. $46.7 \%(\mathrm{n}=14)$ underwent a bedside open tracheostomy, 16.7\% $(\mathrm{n}=5)$ underwent open tracheostomy in a theatre setting, and $36.7 \%(\mathrm{n}=11)$ underwent a percutaneous tracheostomy. Table 1 summarises the patient characteristics in further detail. The mean age of patients undergoing bedside open tracheostomy was 60.5 years old (48-69 years) where majority were male $(\mathrm{n}=10$, $71.4 \%)$ and of Asian ethnicity $(\mathrm{n}=9,64.3 \%)$. The mean duration of mechanical intubation prior to bedside open tracheostomy was 14.5 days. $71.4 \%(\mathrm{n}=10)$ of patients who had undergone bedside open tracheostomy were still alive and had been successfully weaned from mechanical ventilation, with a mean duration of 17 days from tracheostomy to successful decannulation. In-hospital mortality was reported in $28.6 \%(\mathrm{n}=4)$ in the bedside open tracheostomy group, $20 \%(\mathrm{n}=5)$ in open-theatre group, and $18.2 \%(\mathrm{n}=11)$ in percutaneous group. All deaths were attributed to COVID-19 related complications. No significant tracheostomy related complications were reported with bedside open tracheostomy method whilst $20 \%(\mathrm{n}=1)$ and $9.1 \%(\mathrm{n}=1)$ were reported in open-theatre and percutaneous approach respectively. The average operating time (from skin incision to insertion of tracheostomy tube) for bedside tracheostomy in COVID-19 patients was 9 minutes. There were no intra-operative complications reported and minimal blood loss $(<20 \mathrm{mls})$ were achieved in open-bedside approach. In all cases, the operating surgeons used a Half-Face Air Purifying Respirator, visor or goggles, fluid resistant sterile theatre gown, double-gloving and head and shoe covering. There were no healthcare personnel involved that reported acute respiratory COVID-19 infection within two weeks of the procedure. All the Head and Neck surgeons had a negative outcome for COVID-19 antibodies.

\section{Discussion}


With no established superiority of approach and location of tracheostomy procedures from the standpoint of infectious transmission, the choice is determined by balancing the risks to patients and staff, and considering local expertise and resources.

Performance of surgical tracheostomy in theatre requires availability of operating rooms, negative-pressure ventilation, staff and equipment, with the need for multiple disconnection and reconnection of the breathing circuit. Circuit disconnection could potentially lead to impaired oxygenation in the critically-ill patient due to loss of positive end-expiratory pressure (5), and additionally increase exposure risks to additional personnel during patient transfer. A systematic review evaluating the available international guidelines for tracheostomy in COVID-19(6) highlighted the role of bedside tracheostomy in the Intensive Care Unit (ICU) in negative-pressure rooms. However, it is generally recognised that the availability of negative pressure air flow setting both in operating room and ICU is in reality limited and not a usual part of the UK hospital infrastructure. As with many institutions that were severely affected with COVID-19, multiple operating theatre rooms were converted to accommodate the saturation of ITU beds. These logistical factors, together with scarce operating room resources, favoured open surgical tracheostomy to be performed by the bedside in ICU.

The specific challenges of bedside open approach include limited space, need of transfer of surgical equipment and instruments, and suboptimal lighting and positioning. This was made more challenging due to the enhanced PPE and associated impaired communication. We find that in order to overcome these challenges, bedside open tracheostomies in the ICU should be standardised and meticulously planned with participation of highly experienced surgeons, anaesthetist and scrub team

We followed key recommendations in minimising aerosol generation during open tracheostomy in COVID-19 patients including advancing the endotracheal tube distal to proposed site of tracheal window prior to entry, hyperinflation of endotracheal cuff, withholding ventilation at key points and covering operative site with gauze swabs when ventilation recommences $(7)$.

A key approach in performing a safe and swift bedside tracheostomy is to ensure that major bleeding is avoided. Many of the critically ill tracheostomy candidates will be anticoagulated; making haemostasis even more crucial. Common source of bleeding is typically from the anterior jugular veins and from the encountered thyroid gland and its feeding vessels. A pre-operative ultrasound assessment can be considered in conjunction with palpation of the neck particularly in in obese patients or where anatomical landmarks are difficult to assess by palpation. It provides important anatomical information including distance from skin to trachea, identification of vulnerable structures, such as thyroid gland and blood vessels.

We acknowledge and follow the recommendations to limit the use of diathermy. The evidence surrounding risk of aerosolisation from surgical smoke plumes is still not fully understood (8), however transmission is theoretically plausible. Therefore, we opted for vascular clips and and surgical ties when possible and considered diathermy on case-by-case basis; balancing the potential risk of aerosolisation with the risk of intra-operative bleeding. To mitigate theoretical viral transmission from diathermy plumes, we ensured the use of an extractor suction. Our practice also includes the use of LigaSure sealing device in cases where thyroid isthmus division is required. This approach as opposed to traditional clamping, division and ligation with transfixion sutures; is considered a less time-consuming option.

Bedside open tracheostomy demonstrated a short mean procedural time of 9 minutes and with no tracheostomy-related complications. The higher in-hospital mortality and mean time to decannulation may be a reflection of the inherent selection bias in the bedside tracheostomy group as these patients are typically selected due to their comorbidities and higher risk of transfer. Further studies are needed to better understand this association.

\section{Conclusion}

Our experience with bedside open tracheostomy demonstrated a short mean procedural time, with no tracheostomy-related complications and no reported viral transmission amongst the healthcare members 
involved. We acknowledge the technical challenges that are associated with operating outside theatre environment, however with careful planning and training, bedside approach to tracheostomy can be considered a more effective and safe approach in the COVID-19 pandemic.

Conflict of interests: None declared

\section{References}

1. Tran K, Cimon K, Severn M, Pessoa-Silva CL, Conly J. Aerosol generating procedures and risk of transmission of acute respiratory infections to healthcare workers: a systematic review. PLoS One. 2012;7(4):e35797. doi:10.1371/journal.pone.0035797. pmid:http://www.ncbi.nlm.nih.gov/pubmed/22563403

2. McGrath BA, Brenner MJ, Warrillow SJ, Pandian V, Arora A, Cameron TS, et al. Tracheostomy in the COVID-19 era: global and multidisciplinary guidance. Lancet Respir Med. 2020. https://www.thelancet.com/journals/lanres/article/PIIS2213-2600(20)30230-7/fulltext

3. Jacob $\mathrm{T}$ et al. COVID Tracheostomy guidance. 2020. http://www.tracheostomy.org.uk/storage/files/ENT_UK\%20COVID\%20tracheostomy\%20guidance_compressed.pdf

4. COVID-19 tracheostomy guideline. British Laryngology Association. 2020. https://www.britishlaryngological.org/sites/default/files/BLA\%20Tracheostomy\%20guideline\%20BLA\%20April\%202020\%20FINAL.pdf

5. Lew TWK, Kwek TK, Tai D, Earnest A, Loo S, Singh K, Kwan KM, Chan Y, Yim CF, Bek SL, Kor AC, Yap WS, Chelliah R, Lai YC, Goh SK: Acute respiratory distress syndrome in critically ill patients with SARS. JAMA 2003; 290:374-80

6. Chiesa-Estomba CM, Lechien JR, Calvo-Henríquez C, et al. Systematic review of international guidelines for tracheostomy in COVID-19 patients [published online ahead of print, 2020 Jun 5]. Oral Oncol. 2020;108:104844. doi:10.1016/j.oraloncology.2020.104844

7. McGrath BA, Ashby N, Birchall M, et al. Multidisciplinary guidance for safe tracheostomy care during the COVID-19 pandemic: the NHS National Patient Safety Improvement Programme (NatPatSIP) [published online ahead of print, 2020 May 12]. Anaesthesia. 2020;10.1111/anae.15120. doi:10.1111/anae.15120 Mowbray NG, Ansell J, Horwood J, Cornish J, Rizkallah P, Parker A et al. Safe management of surgical smoke in the age of COVID-19. Br J Surg 2020
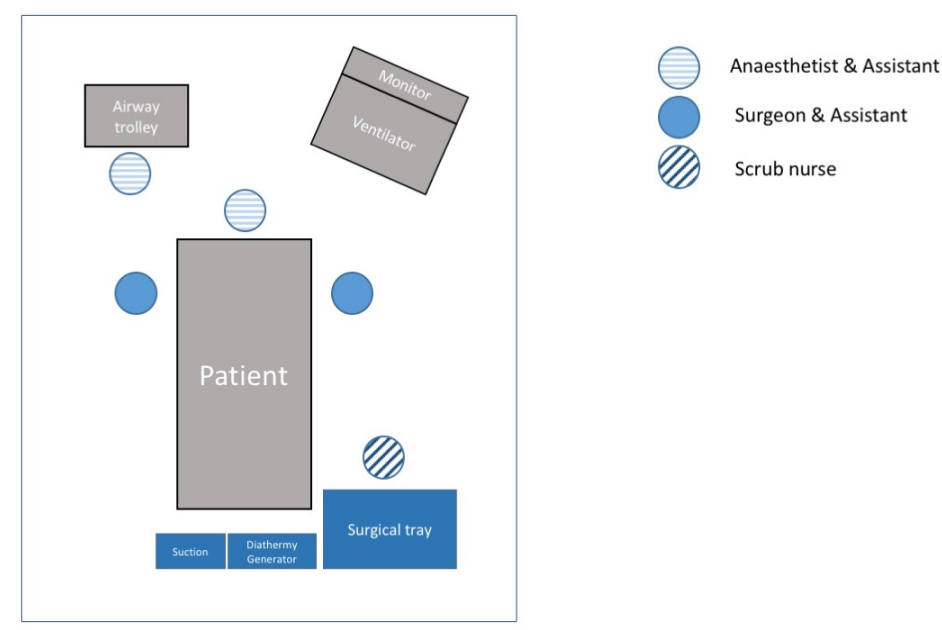
COVID-19

\section{Bedside Surgical Tracheostomy}

\begin{tabular}{|c|}
\hline $\begin{array}{l}\qquad \text { Indication } \\
\text { Facilitation and weaning from } \\
\text { prolonged mechanical ventilation }\end{array}$ \\
\hline $\begin{array}{l}\quad \text { Timing } \\
\text { Preferably delayed until after } 10 \text { days of } \\
\text { mechanical ventilation }\end{array}$ \\
\hline \begin{tabular}{ll} 
& \multicolumn{1}{c}{ Team } \\
- & 2 Consultant ENT surgeons \\
- & 1 Consultant Anaesthetist \\
- & 1 Anaesthetic assistant \\
- & 1 Scrub nurse \\
- & 1 Standby nursing staff
\end{tabular} \\
\hline
\end{tabular}

Team Briefing

- Airway management briefing critical to procedure

- Anaesthetic consideration: full paralysis to reduce risk of cough

\section{Equipment}

- Personal Protective Equipment (PPE)

- Surgical headlight : key requirement to compensate for suboptimal

- Portable Ultrasound machine: consider bedside ultrasound imaging for assessment of thyroid isthmus

- Tracheostomy surgical tray including vascular clips and surgical ties

- Bipolar diathermy with smoke evacuator

- Ligasure sealing device: used if division of thyroid isthmus is required

- Tracheostomy tube: a range of cuffed, nonfenestrated tubes - aim for size 8 in males and size 6 for females. The tracheostomy tube should be tested prior to starting the procedure to ensure that the cuff is patent with no air leak

- Heat Moisture Exchange (HME) viral filter: to provide humidification and prevents generation of aerosols

\begin{tabular}{|l|}
\hline \multicolumn{1}{|c|}{ Patient } \\
- $\quad$ Ponfirm patient as per WHO checklist \\
- Skin preparation and drape
\end{tabular}

Pre-tracheal entry
- - Maintenance of bloodless field
- Haemostasis: Use of vascular clips and surgical ties. Minimal use of diathermy.
- Consider use of LigaSure sealing device for isthmus manipulation

Pause
- Anaesthetist informed prior to tracheal entry
- $\quad$ Confirms full paralysis and a closed ventilation circuit
(APL) valve, allow passive expiration, consider clamping ETT
(APL) valve, allow passive expiration, consider clamping ETT

\section{Advance Endotracheal Tube (ETT)}

- Deflate ETT cuff and advanced beyond proposed tracheal window

- Hyperinflate ETT cuff and re-establish oxygenation with positive end-expiratory pressure (PEEP)

- Prior to tracheal entry: Cease ventilation, turn off flows with open APL valve, allow passive expiration, consider clamping ETT

Create tracheal window

\section{Tracheal entry}

- Deflate ETT cuff and withdraw proximal to tracheal window

- Insert tracheostomy tube and inflate tracheostomy tube cuff, introducer replaced with non fenestrated inner tube and HME viral filter

- Connect tracheostomy to circuit, resume and confirm ventilation with end tidal $\mathrm{CO} 2$

- Cover operative site with gauze swabs once ventilation recommences

- Secure Tracheostomy with sutures, tape and dressing Withdraw clamped ETT carefully 


\begin{tabular}{|c|c|c|c|c|c|c|c|}
\hline \multirow{3}{*}{\multicolumn{2}{|c|}{ Patient Characteristics }} & \multicolumn{6}{|c|}{ Tracheostomy Approach } \\
\hline & & \multicolumn{2}{|c|}{$\frac{\text { Open-Bedside }}{(n=14)}$} & \multicolumn{2}{|c|}{$\frac{\text { Open-Theatre }}{(n=5)}$} & \multicolumn{2}{|c|}{$\frac{\text { Percutaneous }}{(n=11)}$} \\
\hline & & No. & (value) & No. & (value) & No. & (value) \\
\hline \multicolumn{2}{|c|}{ Age, mean (range) } & & $60.5(48-69)$ & & $54.6(46-66)$ & 6 & $62.1(48-77)$ \\
\hline \multirow[t]{2}{*}{ Gender } & Male & 10 & 71.4 & 1 & 20 & 8 & 72.7 \\
\hline & Female & 4 & 28.6 & 4 & 80 & 3 & 27.3 \\
\hline \multirow[t]{4}{*}{ Ethnicity } & White British & 4 & 28.6 & 1 & 20 & 8 & 72.7 \\
\hline & White Other & 0 & 0 & 2 & 40 & 0 & 0 \\
\hline & Asian British & 9 & 64.3 & 2 & 40 & 2 & 18.2 \\
\hline & Black/British Caribbean & 1 & 7.1 & - & - & 1 & 9.1 \\
\hline \multicolumn{2}{|c|}{$\begin{array}{l}\text { Mean time from intubation to } \\
\text { tracheostomy (days) }\end{array}$} & & 14.5 & & 11.40 & & 14.27 \\
\hline \multicolumn{2}{|c|}{$\begin{array}{l}\text { Mean time from tracheostomy to } \\
\text { decannulation (days) }\end{array}$} & & 17 & & 12.3 & & 15.4 \\
\hline \multicolumn{2}{|c|}{ Inpatient Mortality (\%) } & 4 & 28.6 & 1 & 20 & 2 & 18.2 \\
\hline \multicolumn{2}{|c|}{ Mean duration of procedure (mins) } & & 9 & & 31 & & * \\
\hline \multicolumn{2}{|c|}{ Tracheostomy complications (\%) } & 0 & 0 & 1 & 20 & 1 & 9.1 \\
\hline
\end{tabular}

